\title{
Illusory figures: Individual differences in apparent depth and lightness
}

\author{
THEODORE E. PARKS \\ University of California, Davis, California \\ and \\ WILLIAM MARKS \\ University of Michigan, Ann Arbor, Michigan
}

\begin{abstract}
When the luminance of a pattern that induces a sharp-edged illusory circle was decreased, both illusory contrast with the surround and the illusory depth difference between that circular area and the inducing elements reliably increased. Thus, changes in both of these effects apparently participate in (contribute to) the increased overall salience that had previously been found with decreased luminance. However, among individuals, the correlation between this improvement in contrast and this increase in depth was exceedingly small.
\end{abstract}

As is well known, in a typical illusory figure, part of a homogeneous area appears to both (1) differ in lightness from its surround and (2) be closer in depth than that surround (see, e.g., Figure 1). Ever since the pioneering proposals of Coren (1972), there has been a great deal of experimental investigation (cf. Parks, 1984), as well as theoretical speculation (cf. Pritchard \& Warm, 1983), concerning the relationship between these two aspects of illusory figures. The present study focuses upon a particular possibility: that illusory lightness gradients and illusory depth are alternative means by which the system expresses an illusory contour.

That illusory lightness and illusory depth differences are alternatives to one another, at least among various displays, is already clear from demonstrations of illusory figures that do not exhibit an illusory lightness effect (e.g., Parks, 1980; Prazdny, 1983) and illusory figures that do not include a depth effect (e.g., that seen in the freestanding display of Ware \& Kennedy, 1978; but see also Rock \& Anson, 1979). The present research concerns, instead, the possibility that even when both illusory properties occur within a single display (and so are not alternatives in a strict "either/or" sense), they may still be alternatives to the extent that any variation in the overall salience of an illusory figure may be expressed as a variable mix of variation in depth and variation in lightness.

To expand, the suggestion to be considered is that, as a final step in the basic process of segmenting the visual array, illusory modifications are made in lightness and depth which vary directly with the decisiveness of that segmentation, but since the two are alternatives, large

Reprint requests should be addressed to the first author at the Department of Psychology, University of California, Davis, California 95616. changes in one are not necessarily accompanied by corresponding changes in the other.

Indeed, Halpern (1981) did find only relatively low correlations between reported lightness and depth effects (the largest being .396) across subjects under a single viewing condition. However, the expected independence of lightness and depth effects would be established more convincingly if it could also be shown that changes in one of these two effects could occur within subjects independently of changes in the other. Specifically, the present research examined the degree to which reducing the illumination incident upon an inducing pattern-a manipula-

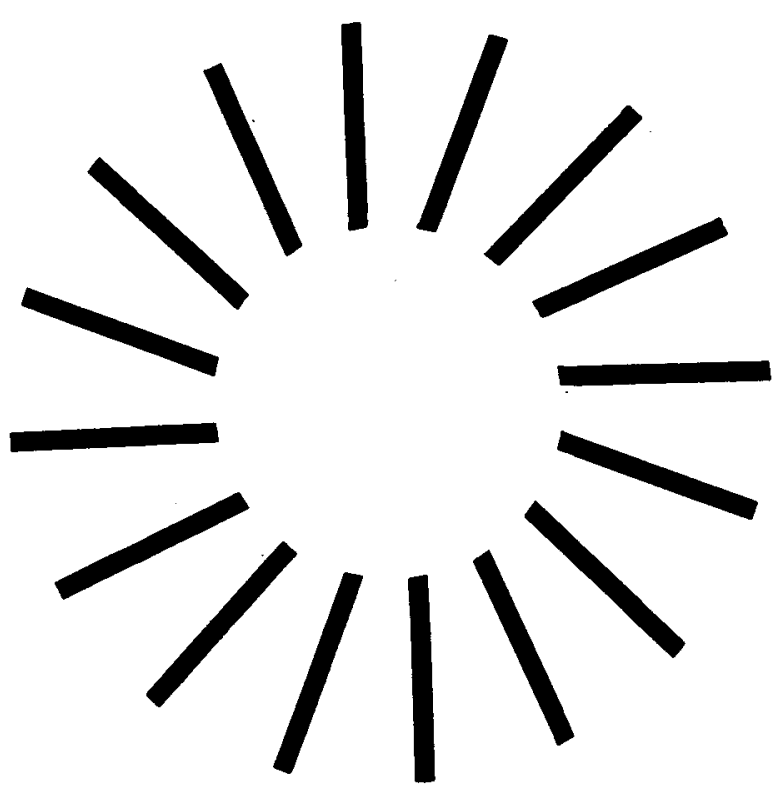

Figure 1. The inducing pattern used in the present experiment. 
tion that is known to increase the overall salience of an illusory figure (Dumais \& Bradley, 1976; Parks \& Marks, 1983) - would increase its apparent lightness contrast and its apparent depth. More to the point, the relationship (or lack of one) between variation in these two aspects of the overall effect could then be explored.

\section{METHOD}

\section{Subjects, Materials, and Apparatus}

A total of 40 naive volunteers were individually shown copies of Figure 1 in an Iconix 6137 tachistoscope. Each pattern was $11 \mathrm{~cm}$ in height and was presented at a distance of $86 \mathrm{~cm}$. This pattern was a good one for present purposes, since it was already known that the salience of the illusion seen in this pattern varied reliably with the level of illumnation (Parks \& Marks, 1983). Since the reflectance of all materials was constant, changes in the level of illumination will hereafter be referred to as changes in luminance.

\section{Procedure}

Each subject first received general instructions that included presentations of stimulus patterns that produced a triangular illusory figure and that indicated that he or she would be shown patterns "like the ones you just saw" under a "variety of conditions." More specifically, the subject was told that each trial would consist of the presentation of a pattern accompanied by a capital $A$ in the lower right-hand corner of the viewing screen, followed by an unmarked pattern, then by the marked pattern again, and, finally, by a last presentation of the unmarked pattern. Each presentation of either pattern was $2.8 \mathrm{sec}$ in duration and was separated from the next presentation by a blank field $.2 \mathrm{sec}$ in duration. Previous work (Parks \& Marks, 1983) had shown that this pacing allowed subjects a comfortable chance to view each pattern, and this interpattern interval made the onset of succeeding patterns more pleasant. Since the experimenter waited for (and recorded) the subject's response before initiating the next trial, the intertrial interval varied, but was on the order of several seconds.

Except for the letter that marked one of them, the two patterns presented on any trial were always identical (two copies of Figure 1). However, the luminance of the marked pattern was always $25 \mathrm{~cd} / \mathrm{m}^{2}$, whereas that of the unmarked pattern varied among $2.6,3.6,7.2$, and $32.0 \mathrm{~cd} / \mathrm{m}^{2}$. These are all pleasantly low levels and were found to be effective with respect to salience in previous work (Parks \& Marks, 1983). The luminance of the interstimulus field (which also served as an intertrial field) was set at $18 \mathrm{~cd} / \mathrm{m}^{2}$.

On any given trial, the subject was asked to report on either, but not both, (1) the apparent depth of the illusory circle or (2) its apparent lightness, in each case the judgment being relative to the surround. The former responses were made in a relatively straightforward manner: the subject was asked to draw a vertical line through a horizontal line which was already marked with a crosshatch at its midpoint and, specifically, to do so at a distance from that cross-hatch that corresponded to the perceived distance between the illusory circle and the bars in the unmarked pattern. The subject was told (and a diagram on the data sheet reminded him or her) that the distance marked off should be to the right if the circle appeared to be nearer and to the left if farther away. It will be noted that the " $\mathrm{A}$ " pattern is irrelevant to this task, and the subject was so informed.

Assessing lightness effects was somewhat more complicated. First of all, the subject was told that the important thing to notice was how light the circle appeared relative to the paper around it and not simply how light it looked. To reinforce this concept, the subject was then shown a display of three plain circles of increasing real lightness. Each circle was surrounded by a shade of gray that was slightly (but noticeably) darker than that circle. However, by deliberate arrangement, the difference between inside and surround was noticeably less for the lightest and darkest circle. This was done to avoid suggesting that either extreme in circle-lightness could necessarily be the basis for a correct response. All of this was pointed out to the subject with the explanation that "the important thing is that [you must] do something more difficult than just noticing how white the circle is."

The subject was asked to rate the perceived lightness difference seen in the unmarked pattern on a scale of 0 to 100 , where 50 corresponded to the lightness difference seen in the marked pattern.

It will be noted that the tasks involved in assessing the illusory lightness effect and the illusory depth effects were quite different, but, as has been pointed out (e.g., by Halpern, 1981), such diversity is desirable, since intertask similarities may lead to artifactually high correlations between the results obtained for the two tasks.

\section{Design}

Each subject participated in a total of 48 trials divided into 4 blocks of 12 trials each. Half of the subjects began with a block on which depth was judged, and half did so with a block on lightness; blocks thereafter were alternated. Within any block, there were 3 trials at each of the four illumination levels. Specifically, the first 4 trials of any block consisted of 1 trial at each level of luminance; there order was that of one row of a $4 \times 4$ Latin square, all rows of which were employed equally for the first block across subjects within each group. This sequence was repeated three times to yield a 12 trial block. Each succeeding block employed the next succeeding row of the Latin square. Prior to the experimental trials, each subject particpated in 8 practice trials corresponding to the first 4 trials of that subject's first experimental block and the first 4 trials of his or her second block.

\section{RESULTS AND DISCUSSION}

When the data of all subjects are combined, illusory lightness-gradient ratings and the absolute magnitude of depth reports both increased as luminance decreased (see Figure 2). For the former, mean ratings went from 49 for the brightest display to 55 for the dimmest-a small, but reliable, change $[\mathrm{F}(3,117)=14.49, \mathrm{p}<.01]$ paralleling the change in "salience" found previously under the same conditions (Parks \& Marks, 1983). A similar, but relatively larger, improvement was found in reports of apparent depth, mean values going from 3.3 to $5.5 \mathrm{~mm}$ $[F(3,117)=18.62, p<.01]$. It is interesting to note that, for both depth and lightness, 32 of the 40 subjects (but not necessarily the same subjects) reported a larger effect in the dimmest condition than in the brightest.

The really important point, however, emerged when individual differences within these trends were examined: for individuals, increases in one illusory aspect were not necessarily accompanied by proportionate increases in the other. Thus, for these 40 subjects, the correlation between the difference in a subject's ratings for the lightness effect between the two extreme luminance conditions and the difference between depth reports for those same two conditions was small $[\mathrm{r}(38)=.287, \mathrm{p} \cong .10]$. In fact, even that small correlation was largely the product of the results obtained for one subject, who reported a large change in the lightness effect (going from 42 to 63) and also a large and, in fact, aberrant change in the depth effect (going from a rather typical report of $3.5 \mathrm{~mm}$ in the worst condition to a very unusual report of $17.4 \mathrm{~mm}$ in the best). Since this difference $(13.9 \mathrm{~mm})$ is more than 2 SDs greater than the next largest difference found for 


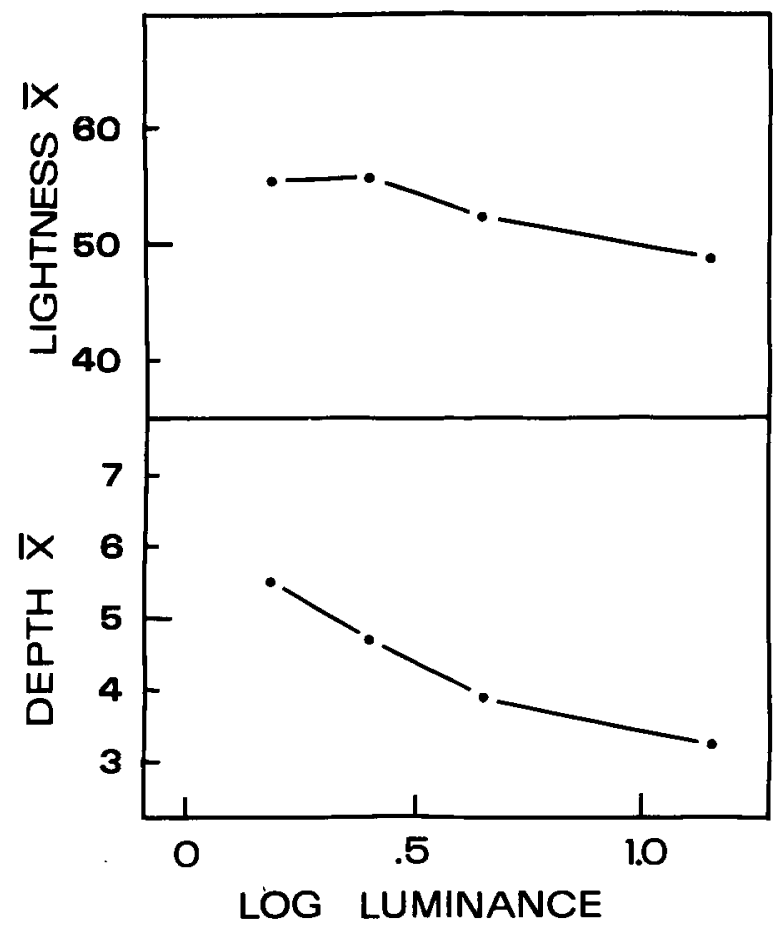

Figure 2. The mean ratings given to illusory lightness contrast (top panel) and mean depth reports (low panel) as a function of luminance relative to $1.8 \mathrm{~cd} / \mathrm{m}^{2}$ (the lowest value used in Parks \& Marks, 1983). Depth values are given in millimeters.

any subject, it was worthwhile to recompute the overall correlation excluding this observer. In fact, for the remaining 39 subjects, that correlation accounts for less than $4 \%$ of the variance $[\mathrm{r}(37)=.190]$. Another subject was distinctly aberrant in a different way. This subject was the only one of 40 for whom both the depth effect and the lightness effect decreased (rather than increased) as illumination was reduced. Furthermore, in a written answer to postexperimental questioning, this subject reported that, contary to the instructions, "I gave large numbers when the circle's darkness or lightness on the inside seemed greater than the outside's light or darkness." Without this subject, the correlation drops virtually to zero $[\mathrm{r}(36)=.060]$, accounting for less than $1 \%$ of the variance.

These low correlations cannot be attributed to the fact that the range of depth reports (and, therefore, of difference scores for depth) was small. For one thing, although absolute difference scores are entered into the computation, each correlation coefficient reflects a relationship between $\mathrm{z}$ scores rather than between absolute values. Thus, except in the special case (which is not the case here) of trying to estimate the correlation for a whole population when only part of the variability of that whole has been sampled (McNemar, 1962), range is irrelevant. Furthermore, by one measure of variability, the coefficient of variation (cf. Sokal \& Rohlf, 1981), the difference scores for depth were actually slightly more vari- able than those obtained for lightness ratings, and both were reasonably large, the values being $47 \%$ and $39 \%$, respectively. Furthermore, the present low correlations cannot be attributed to the difficulty of the rating task used to assess the lightness effect. It is true that had that task been difficult enough to elicit random responses, low correlations (like those obtained here) would have resulted, but the reliable differences found between illumination conditions show that it did not do so to any large extent.

It is, of course, axiomatic that the null hypothesis cannot be proven. Additionally, it must be recognized that the use of a rating technique, such as that used to assess illusory contrast, leads to the possibility that individual differences in the interpretation of the scale employed might tend to obscure an underlying phenomenal relationship between the dimension thus measured and any other that is differently measured. Nevertheless, it is also clear that, given the large sample used here, such low values as any of those found here suggest a profoundly small relationship between the present dependent variables. Clearly, many subjects reported a relatively large increase in one dimension while doing the most that could be done to communicate a very small improvement in the other. For instance, it is difficult to imagine any interpretation of the rating scale that would lead a subject who reported a depth effect that was half again as large (or more) and who supposedly experienced a correspondingly large change in the contrast effect to assign a number to the latter which was only two or three units greater than the standard, " 50 ." Yet, precisely that happened often.

In addition to this major finding, other secondary (albeit interesting) points emerged. One concerns the direction of the depth effect rather than its absolute size. That is, far more reports were obtained of an illusory surface which seemed to lie behind a circular opening within the inducing pattern than would be expected from previous research (e.g., Halpern, 1981). To be specific, such " window" reports were obtained on $30 \%, 28 \%, 35 \%$, and $35 \%$ of all trials for the brightest through dimmest conditions, respectively. Furthermore, and importantly, such a depth effect seems to have been unrelated to the illusory lightness effect. For example, within the best condition, those subjects who reported a "window" effect on all six trials at that level $(\mathrm{N}=2)$ gave a mean lightness rating of 55.6. For those who never reported a "window" $(\mathrm{N}=12)$, the mean was a very similar 54.8 . In other words, both extreme sorts of subjects reported a lightness effect that was extremely close to the overall mean obtained in that condition.

Such reversals of the usual depth effect make two suggestions. First, to say that a depth effect is typical cannot be taken to mean that apparent occlusion of parts of the inducing elements is the rule (since apparent omission often occurs instead). Second, the similarity in lightness reports between observers who experienced this reversal and those who reported the usual arrangment in depth sug- 
gests that some source other than figure-ground lightness contrast (e.g., Parks, Rock, \& Anson, 1983) must play an important role in this particular pattern. That is, in his classic reversible bunny/faces demonstation, Coren (1969) showed that when part of a white homogeneous field is seen as figure, it will be lightened illusorily so as to contrast more with its surround, but when that same part is seen as ground, no such illusory lightening occurs. Here, however, the same illusory lightness difference occurs between the circular area and its surround (and to the same extent) whether the circle appears as figure or as part of a mostly occluded background. Altenatively, given the regular spacing of the repeated bar elements in the present pattern, assimilation of darkness between the bars (cf. Day \& Jory, 1978) would seem to be a likely candidate for the illusory lightness effect seen here.

As a final observation, reports that the illusory circle appeared to be at the same depth as the bars occurred on approximately $13 \%$ of all trials, a figure that agrees quite closely with Halpern's (1981) finding of such reports on $16 \%$ of her trials. There is, however, one difficulty in interpreting this finding. That is, the four subjects who reported no depth on at least two trials at the lowest level of luminance (the most such reports for any subject in that condition) gave a mean lightness rating for that condition of only 46, while the value of 55 obtained overall. Such low ratings-lower, in fact, than the value assigned to the standard-might be the result of these observers' attempting to indicate that occasionally no illusory figure occurred at all (or, at best, a very poor one). Alternatively, it may be that the illusory figure appears to be so thin and so close to the inducing elements that subjects feel that that depth would be exaggerated if they drew even the thickness of a pencil-line alongside the hash mark as close as mechanically possible to it. As a result of such uncertainties, reports of no depth (i.e., of coplanearity of the illusory circle with the inducing bars) are probably for now best considerd as a tantilizing, but only possible, parallel to the effect more often seen in free-standing displays (e.g., Ware \& Kennedy, 1978, Figure 2).

\section{CONCLUSION}

To return to the result of greatest importance, at least for the present pattern (one that is optimal for assimilation effects; Jory \& Day, 1979) changes in the absolute value of the illusory depth effect seem to be not at all tightly linked to changes in the magnitude of the illusory lightness gradient or vice versa. It is as though the visual system, having determined that part of what would otherwise appear to be a uniform surface is separate, introduces illusory alterations in lightness and depth as a means of expressing that decision. Appropriately enough, the more certain that decision (or, to be more precise, the less available any contary evidence to that decision; Rock \& Anson, 1979), the greater are the alterations that are made to express it and, therefore, the greater the weight given those illusory contours for the benefit of further visual processing (e.g., pattern recognition). However, it appears that illusory lightness and illusory depth are somewhat redundant for that purpose, so that large individual differences occur in the way that an increase in salience is expressed. The point of such theorizing is not, of course, to predict large individual differences; they would be expected under virtually any theory. The point, rather, is to suggest an understanding of why such individual differences can be tolerated.

\section{REFERENCES}

COREN, S. (1969). Brightness contrast as a function of figure-ground relations. Journal of Experimental Psychology, 80, 517-524.

Coren, S. (1972). Subjective contours and apparent depth. Psychological Review, 79, 359-367.

DAY, R. H., \&ORY, M. K. (1978). Subjective contours, visual acuity, and line contrast. In J. C. Armington, J. E. Krauskopf, \& B. Wooten (Eds.), Visual psychophysics and physiology (pp. 331-340). New York: Academic Press.

Dumais, S. T., \& Bradley, D. R. (1976). The effects of illumination level and retinal size on the apparent stength of subjective contours. Perception \& Psychophysics, 19, 339-345.

HALPERN, D. F. (1981). The determinants of illusory-contour perception. Perception, 10, 199-213.

JoRY, M. K., \& DAY, R. H. (1979). The relationship between brightness contrast and illusory contours. Perception, 8, 3-9.

McNemar, Q. (1962). Psychological statistics (3rd ed.). New York: Wiley.

Parks, T. E. (1980). [Letter to the Editor.] Perception, 9, 723.

PARKs, T. E. (1984). Illusory figures: A (mostly) atheoretical review. Psychological Bulletin, 95, 282-300.

PARKs, T. E., \& MARKs, W. (1983). Sharp-edged vs. diffuse illusory circles: The effects of varying luminance. Perception \& Psychophysics, 33, 172-176.

Parks, T. E., Rock, I., \& Anson, R. (1983). Illusory contour lightness: A neglected possibility. Perception, 12, 43-47.

Prazdny, K. (1983). Illusory contours are not caused by simultaneous brightness contrast. Perception \& Psychophysics, 34, 403-404.

Pritchard, W. S., \& Warm, J. S. (1983). Attentional processing and the subjective contour illusion. Journal of Experimental Psychology: General, 112, 145-175.

Rock, I., \& ANSON, R. (1979). Illusory contours as the solution to a problem. Perception, 8, 665-681.

SoKal, R. R., \& RohlF, F. J. (1981). Biometry (2nd ed.). San Francisco: Freeman.

Ware, C., \& Kennedy, J. M. (1978). Perception of subjective lines, surfaces, and volumes in 3-dimensional constructions. Leonardo, 11, 111-114.

(Manuscript received December 26, 1984; revision accepted for publication May 15,1985 .) 